\title{
Fabrication of hollow maxillary complete denture: A simplified technique
}

\author{
Jogeswar Barman ${ }^{1}$, Rubaiya Rahman ${ }^{2 *}$, Sumeet Bhattacherjee ${ }^{3}$ \\ ${ }^{1}$ Reader, ${ }^{2,3}$ PG Trainee, Dept. of Prosthodontics, Regional Dental College, Guwahati, Assam, India
}

*Corresponding Author: Rubaiya Rahman

Email: rubyripti88@gmail.com

\begin{abstract}
Residual ridge resorption is the reduction in size of the bony ridge under the mucoperiosteum. Prosthetic rehabilitation of severely atrophic ridges has always been difficult for the clinician due to decreased retention, stability and support. Because of such severe resorption the restorative space between maxillary and mandibular residual ridges is increased. Rehabilitation in such cases may result in increased height and weight of the prosthesis further compromising its retention and stability. This in turn overloads the underlying hard and soft tissues that cause further ridge resorption so, in order to keep down this undesirable sequence, the weight of the prosthesis needs to be reduced which can be achieved by making hollow prosthesis. This article describes a simple technique of fabricating a hollow maxillary complete denture.
\end{abstract}

Keywords: Hollow denture, Inter-ridge distance, Simple technique.

\section{Introduction}

Severe resorption of the edentulous maxillae and mandible are generally associated with difficulty in rehabilitation. These may be due to decreased supporting area which leads to narrower, constricted residual ridge as resorption progresses, resulting in large inter-ridge restorative space. The result is a heavy maxillary complete denture with decreased retention and resistance.

Various studies showed gravity and the additional weight to the mandibular complete denture may aid in prosthesis retention, although not universally accepted ${ }^{1}$. Reducing the weight of a maxillary prosthesis, however, is beneficial when constructing an obturator for the restoration of a large maxillofacial defect. $^{2}$ Historically, weight reduction approaches have been achieved using dental stone ${ }^{2}$, cellophane-wrapped asbestos, ${ }^{3}$ silicone putty, ${ }^{4}$ or modelling clay $^{5}$ during laboratory processing to exclude denture base material from the planned hollow cavity of the prosthesis. The disadvantage of the previous techniques is the tedious retrieval of the three-dimensional spacer especially from the anterior region of the prosthesis between the canines due to the curvature of the arch. Fattore et al. ${ }^{6}$ and Holt ${ }^{4}$ have used another techniques for fabricating a hollow prosthesis. The primary disadvantage of their technique was the junction between the two polymerized portions of the denture were at the borders of the denture which increases the risk of seepage of fluid into the denture cavity. Furthermore, this junction is a common site for post-insertion adjustment increasing the risk of leakage.

The present clinical report describes a simple technique for fabrication of a hollow maxillary complete denture.

\section{Case Report}

A 48-year-old male patient attended OPD of Department of Prosthodontics, Regional Dental College, Guwahati, Assam with chief complaint of difficulty in chewing and aesthetics as his front teeth was not visible during smiling. Extra oral examination revealed patient was having long upper lip with drooped corners of mouth. Intra oral examination revealed severely resorbed edentulous ridges (Fig. 1) with increased inter-ridge distance and the ridges were in edge to edge relationship. On further questionnaire patient stated that he was not satisfied with his previous denture as the teeth was attritted with no incisal visibility while speaking and the denture was under extended. Considering all findings, it was decided to fabricate a hollow maxillary denture with conventional mandibular denture.

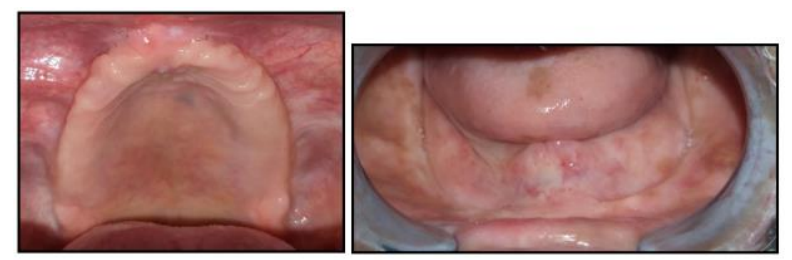

Fig. 1: Intra-oral view

\section{Procedure}

Conventional steps for fabrication of complete denture prosthesis was carried out till the preparation of the master cast. Maxillary occlusal rim was adjusted accordingly to provide proper esthetics. Jaw relation was recorded and tryin was done (Fig. 2). The trial denture was then processed in conventional manner till wax elimination stage. Baseplate wax of $2 \mathrm{~mm}$ thickness was adapted over the master cast and the counterpart leaving an 3D empty space (Fig. 3). A temporary putty spacer (Heraeus Variotime, Kulzer) was fabricated and adjusted till the trial closure to achieve the hollow cavity. The replica of the putty spacer was carved in a glycerine soap (Pears, Mumbai, India) using a Lecron wax carver (API) for use during acrylization (Fig. 4).

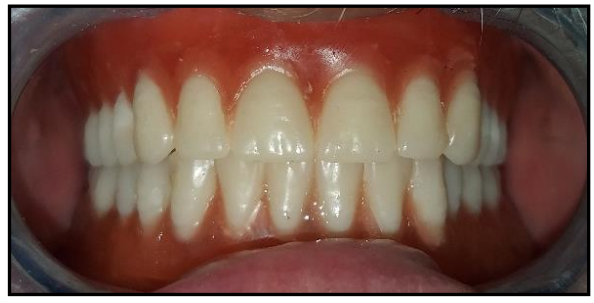

Fig. 2: Try-in stage 


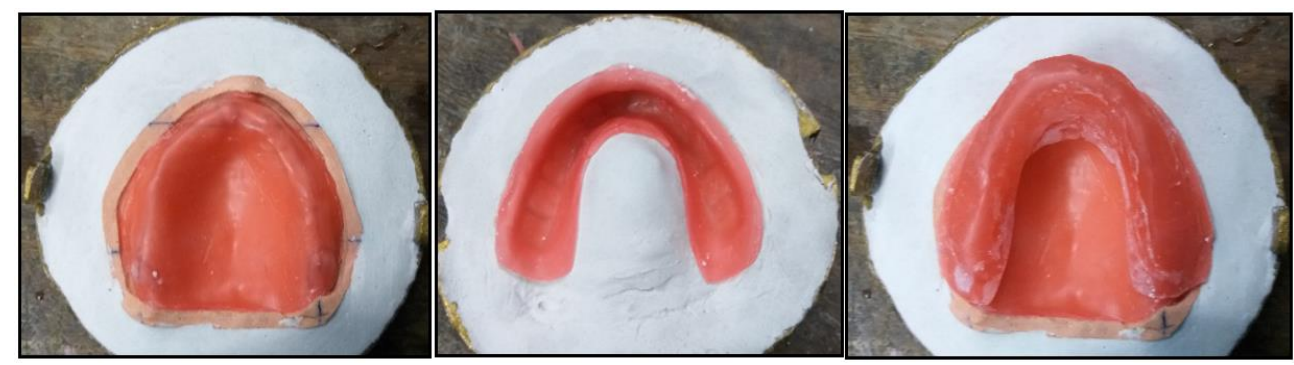

Fig. 3: Adaptation of wax spacer
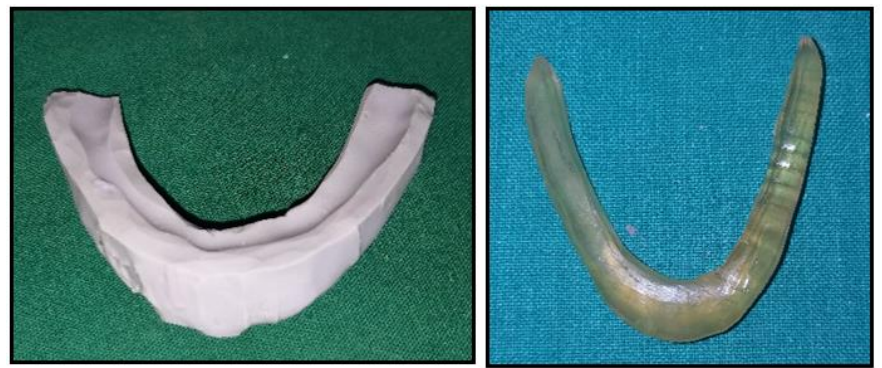

Fig. 4: Putty and soap spacer

The accuracy of the 3D spacer was assessed by placing it between the master cast and its counterpart. After this, a trial closure was carried out using the temporary putty spacer. The flasks were opened and temporary putty spacer retrieved. The mould space was visually assessed for adequate resin thickness all around the hollow cavity. The hollow space left by the temporary putty spacer was now filled with the previously carved soap spacer and the final closure between the components of the flask was achieved. The denture was acrylized with the soap spacer inside it in the conventional manner. The denture was retrieved after processing. Two openings were cut distal to the second molar using a micromotor handpiece, (Fig. 5). The denture was then immersed in a bowl of water to allow the dissolution of soap. Water spray was used to flush traces of soap completely. The hollow cavity was air-dried, and the openings were sealed using auto polymerizing acrylic resin. The denture was immersed in water overnight to assess leakage into the cavity. A water test was performed to evaluate the hollow space as evident by the floating denture (Fig. 6).

Upper and Lower dentures were then finished, polished, and delivered to the patient (Fig. 7). The patient was reviewed after $24 \mathrm{hrs}$, a week and minor adjustments were made.

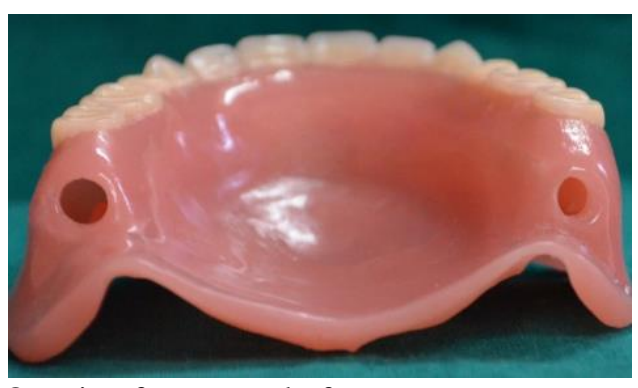

Fig. 5: Opening for removal of spacer

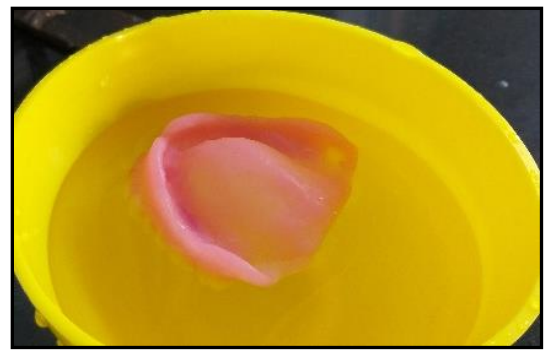

Fig. 6: Floating test of denture

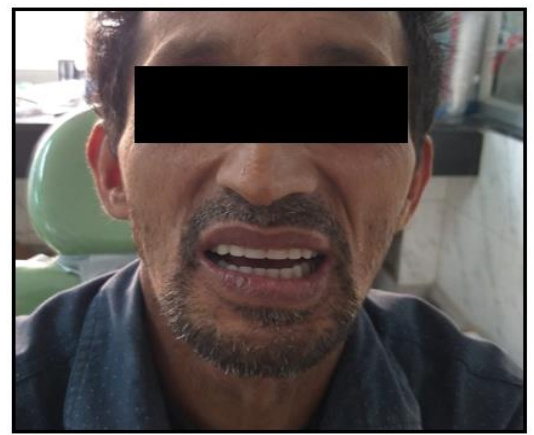

Fig. 7: Insertion of denture

\section{Discussion}

The fundamental goal of prosthodontic rehabilitation is to restore the functional and aesthetic deficiencies. Tooth loss leads to residual ridge resorption which is a complex phenomenon driven by various anatomic, prosthetic, functional and metabolic factors. Extreme resorption of either ridge will lead to a reduced denture-bearing area with large inter-ridge space which in turn will affect retention, stability and support for the complete denture. ${ }^{7}$ Prosthetic rehabilitation in such scenarios often results in increased height and weight of the prosthesis, overloading the residual ridges and further compromising the retention and stability of the prosthesis. 
Fabrication of hollow dentures decreases the weight of the prosthesis which in turn increases retention and stability. ${ }^{2}$ Various solid three-dimensional spacer were used in the literature to reduce the weight of the denture. Salt was also tried as a three-dimensional spacer in some studies, but failed to sustain pressures produced during flask closure resulting inadequate hollow cavity inside the prosthesis. Alternately, the fabrication of separate pieces of the prosthesis was also tried by joining the individual pieces using autopolymerizing acrylic resin. ${ }^{4}$ The major drawback of such techniques is that a junction is formed between the two previously polymerized portions of the denture that increased the risk of fluid seepage into the denture cavity.

Most authors have used a double flask technique for fabrication of hollow dentures. ${ }^{4-7}$ In double flask technique a pair of split dental flasks are used with interchangeable counters. Single flask technique enables the operator to achieve a trial closure with the temporary putty spacer which creates space for the final soap spacer avoiding any extra pressure over it during the final closure. The trial closure also allows the verification of adequate resin thickness all around the spacer or the planned hollow cavity.

The technique described in the presented case used a soap spacer with a high content of glycerine and other humectants in it, rendering it highly water-soluble compared to other soaps. The main advantage of using glycerine soap spacer as documented in other studies are its ability to sustain high curing temperatures (boiling point of glycerine $290^{\circ} \mathrm{C}$ ) and also it doesn't interfere with the polymerization of heat cure acrylic resin leaving no residues inside the hollow cavity. Also because the soap spacer is eventually removed leaving behind a clean hollow cavity, any concern regarding its biocompatibility in the oral cavity is dismissed.

\section{Conclusion}

Hollow maxillary complete denture considerably reduces the weight of the prosthesis which in turn prevents transmission of detrimental forces that would otherwise be transmitted from a conventional heavy prosthesis to the underlying tissues. Thus it helps to preserve underlying tissues and bone. This single flask technique eliminates the requirement of two identical flasks and the extra step of fabrication of a permanent record base. Hence, it's a simple, economical, time-saving and predictable technique.

\section{Source of Funding}

None.

\section{Conflict of Interest}

None.

\section{References}

1. Jacobson TE, Krol AJ. A contemporary review of the factors involved in complete denture retention, stability, and support. Part I: retention. J Prosthet Dent. 1983;49:5-15.

2. el Mahdy AS. Processing a hollow obturator. J Prosthet Dent. 1969;22:682-6.

3. Worley JL, Kniejski ME. A method for controlling the thickness of hollow obturator prostheses. J Prosthet Dent. 1983;50:227-9.

4. Holt RA Jr. A hollow complete lower denture. J Prosthet Dent. 1981;45:452-4.

5. Elliott DJ. The hollow bulb obturator: its fabrication using one denture flask. Quintessence Dent Technol. 1983;7:13-4.

6. Fattore LD, Fine L, Edmonds DC. The hollow denture: an alternative treatment for atrophic maxillae. J Prosthet Dent. 1988;59:514-6.

7. O'Sullivan M, Hansen N, Cronin RJ, Cagna DR. The hollow maxillary complete denture: A modified technique. J Prosthet Dent. 2004;91:591-4.

8. Shetty V, Gali S, Ravindran S. Light weight maxillary complete denture: a case report using a simplified technique with thermocol. J Interdiscip Den. 2011;1:45-8.

9. Chalian VA, Barnett MO. A new technique for constructing a one-piece hollow obturator after partial maxillectomy. $J$ Prosthet Dent. 1972;28:448-53.

How to cite this article: Barman $\mathrm{J}$, Rahman $\mathrm{R}$, Bhattacherjee S. Fabrication of hollow maxillary complete denture: A simplified technique. Int J Oral Health Dent 2020;6(1):63-5. 